\title{
Bidirectional electrostatic linear shuffle motor with two degrees of freedom
}

\author{
E. Sarajlic ${ }^{l}$, E. Berenschot ${ }^{l}$, H. Fujita ${ }^{2}$, G. Krijnen ${ }^{l}$ and M. Elwenspoek ${ }^{I}$ \\ ${ }^{1}$ MESA+ Research Institute, University Of Twente, Enschede, The Netherlands \\ ${ }^{2}$ Institute of Industrial Science (IIS), University of Tokyo, Tokyo, Japan
}

\begin{abstract}
We report on an electrostatic linear inchworm micromotor with two translational degrees-of-freedom. The motor employs built-in mechanical leverage to convert normal deflection of a flexible cross-plate into a small in-plane step and fout electrostatic clamps to enable bidirectional inchworm motion in two orthogonal directions. The motor is realized in a relative simple fabrication process, which combines conventional surface micromachining with trench isolation. Operation in different stepping modes (full, half and diagonal step) accompanied with high overall performance characteristics such as large force $(0.64 \mathrm{mN})$ at moderate voltage ( $45 \mathrm{~V}$ plate and $36 \mathrm{~V}$ clamp), adjustable nanometer-resolution step size $(41 \mathrm{~nm}$ to $63 \mathrm{~nm})$, broad cycling frequency range ( 0 to $80 \mathrm{kHZ}$ ), small dimension (482 $\mu \mathrm{m} \mathrm{x} 482 \mu \mathrm{m}$ ) and large displacement range, makes this inchworm motor an attractive candidate for demanding positioning applications e.g. for (probe based) data storage.
\end{abstract}

\section{INTRODUCTION}

At the $\mathrm{MESA}^{+}$Research Institute work is in progress on a magnetic memory based on scanning probe technology [1]. The memory, named micro Scanning Probe Array Memory ( $\mu$ SPAM), will combine high storage capacity with low power consumption and small size. The basic principle behind $\mu$ SPAM is to scan a discrete magnetic medium by an array of a large number of stationary micromechanical probes (as illustrated in Figure 1) by moving the former in two directions. In contrast to other groups working in the probe storage field [2, 3] a large number of micropositioners with an integrated medium sled is employed to increase concurrency and reduce power consumption.

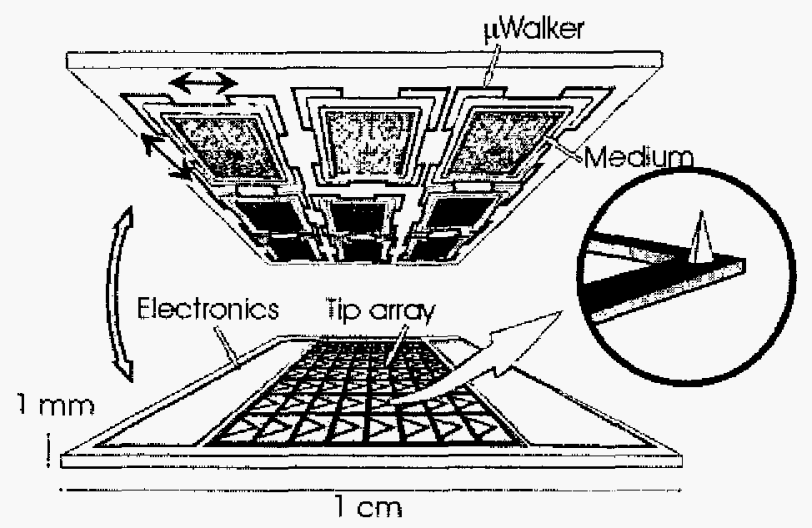

Figure 1: Micro Scanning Probe Array Memory concept (courtesy of L. Abelmann and M. Bolks).

A successful implementation of the $\mu$ SPAM concept requires a high-performance micropositioner, which allows two-axis planar motion with demanding performance such as high maximum speed $(\mathrm{mm} / \mathrm{s}-\mathrm{cm} / \mathrm{s}$ range), high acceleration, large force ( $\mathrm{mN}$ range), high positional accuracy (tens of $\mathrm{nm}$ ) and low power consumption. Shuffle motors [4-6] are promising candidates to perform these demanding tasks due to small size, large displacement range, high speed, high resolution, large force, high reliability and low power consumption.

A shuffle motor is an electrostatic linear inchworm motor, which consists of an elastic plate for the step generation and two voltage-controlled clamps placed at the ends of the plate to enable stepwise motion with large stroke only limited by flexure design. Performance and reliability of the first shuffle motor [4], fabricated by surface micromachining, was limited due to the charge accumulation in an insulating silicon nitride layer. The negative effect of charge accumulation was eliminated in an improved shuffle motor design [5], which was realized in a five-layer polycrystalline silicon surface micromachining process (SUMMiT V). A combination of trench isolation technology and conventional surface mictomachining has significantly simplified the fabrication process and improved the performance and reliability of shuffle motors [6]. However, the operation of shuffle motors, reported until now, was limited to bidirectional motion along a single axis.

We report on the design and fabrication of the first electrostatic shuffle micromotor with two translational degrees-of-freedom (DOF).

\section{WORKING PRINCIPLE}

A schematic view of the 2-DOF shuffle motor with a walking sequence is shown in Figure 1. The motor consists of a flexible cross-plate and four voltage-controlled clamps placed at the ends of the plate. The plate and the clamps are mutually electrically insulated allowing individual biasing of each component. By application of an appropriate voltage actuation sequence a walking motion of the motor is achieved.

The actuation sequence begins with activation of a first clamp (I). Subsequently, a voltage difference is applied between the plate and the grounded substrate causing a downward deflection of the plate. Since the nomal deflection is much larger than the induced shortening of the plate there is a built-in mechanical leverage resulting in a small but powerful longitudinal contraction of the entire motor. The contraction moves the inactive clamps closer to the activated clamp (II). Next, a second clamp on the opposite side is activated (III). The first clamp is released (IV). The released cross-plate stretches and pushes the inactive clamps outward (V). The voltage actuation sequence is completed by activation of all clamps (VI). 


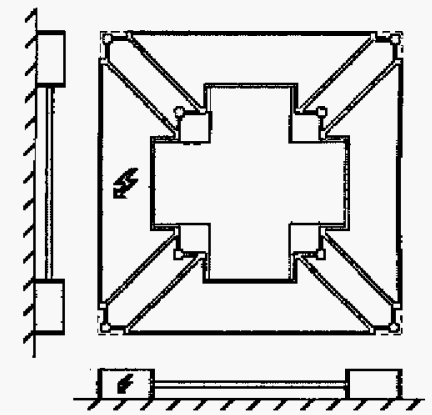

(I)

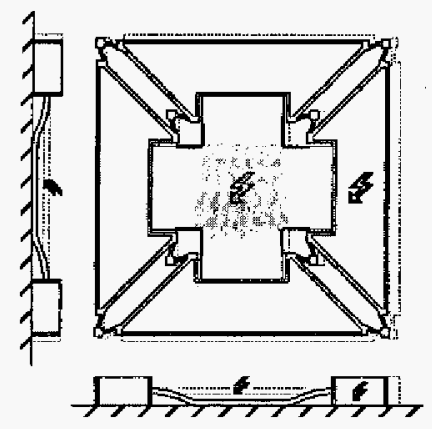

(IV).

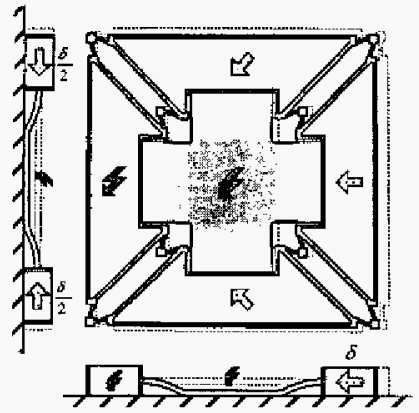

(II)

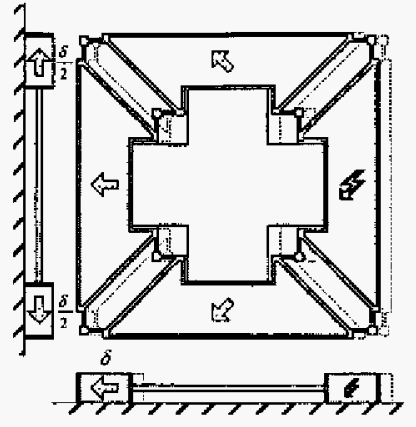

(N)

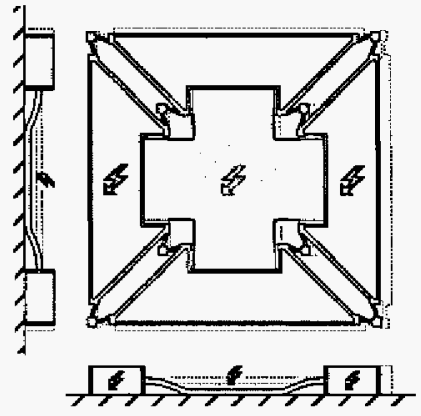

(III)

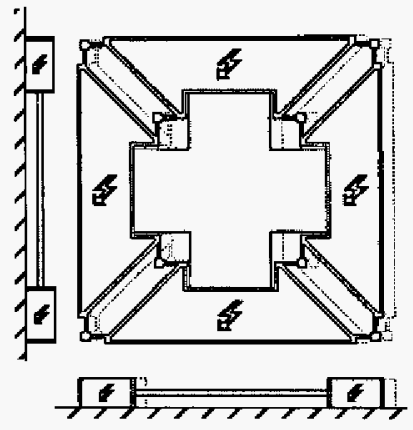

(VI)

Figure 2: Vollage actuation sequence for a single step to the left: (I) Left clamp is activated, (II) Deflection of the cross plate causes a powerful contraction, (III) Right clamp is activated, (IV) Left clamp is released, (V) Stretching of the cross plate moves the clamps, (VI) All clamps activated.

At the end of the sequence, the whole actuator is moved by a single step and is ready to make another step in a wanted direction: A large displacement along two orthogonal axes can be achieved by adding successively a large number of steps. The motor can be operated in different stepping modes by a simple adjustment of the voltage actuation sequence. Actuation sequences for a full, half and diagonal step are shown in Figure 3.
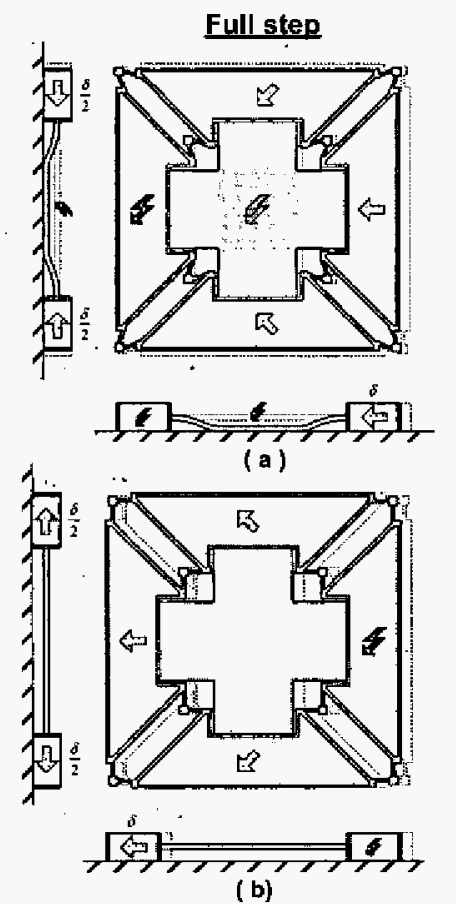
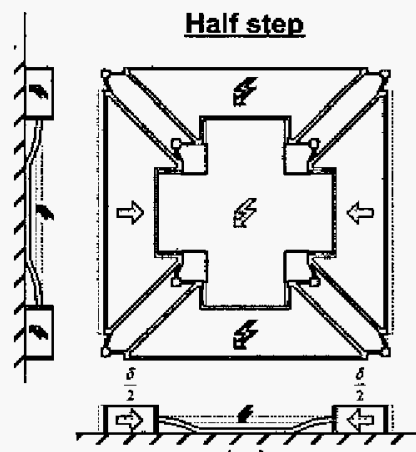

(a)

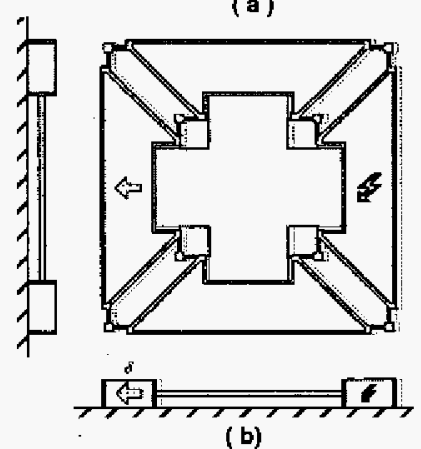

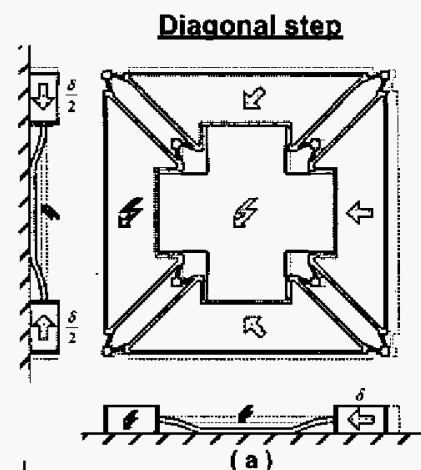

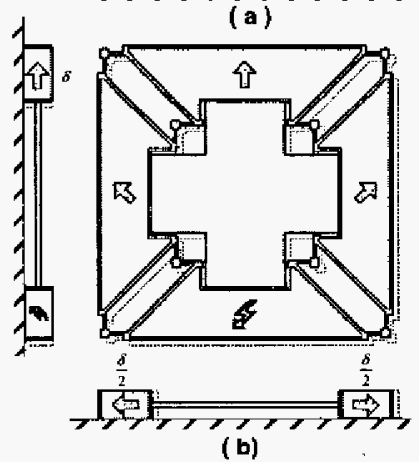

Figure 3: A change in the actuation voltage sequence restlts in different stepping modes: full step (left), half step (center), diagonal step (right). 


\section{FABRICATION}

Vertical trench isolation [7] combined with a conventional surface micromachining process is used to fabricate the proposed 2-DOF shuffle motor. A relative simple fabrication process, illustrated in Figure 4, employs only two polycrystalline silicon device layers and four photolithography masks.

Silicon oxide $\quad \square$ Silicon nitride $\square$ (Poly)silicon

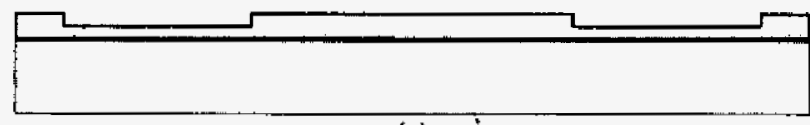

(a)

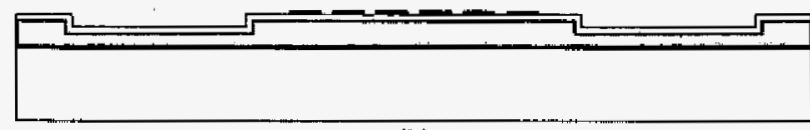

(b)

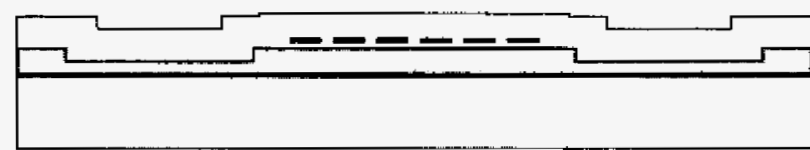

(c)

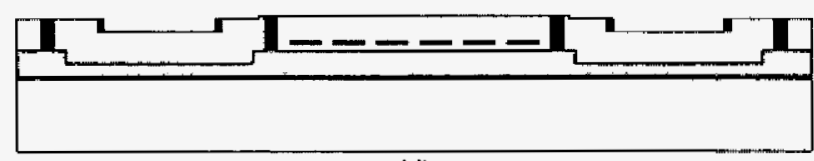

(d)
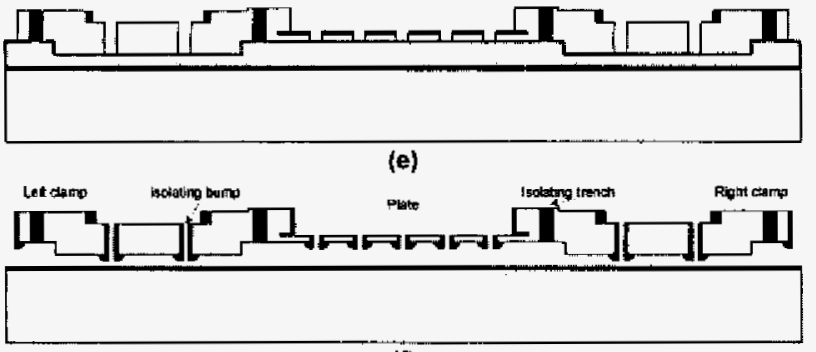

(f)

Figure 4: Condensed fabrication sequence of $2 D O F$ shuffle motor.

The fabrication process starts with the deposition of a 210 $\mathrm{nm}$ low-stress L.PCVD silicon nitride layer on a highly conductive silicon substrate. On the coated substrate, a first $1.5 \mu \mathrm{m}$ PECVD silicon oxide layer is deposited. Molds for the clamps are defined by an isotropic etching of this layer in BHF solution followed by a deposition of a second 0.5 $\mu \mathrm{m}$ PECVD silicon oxide layer (a). On the patterned surface, a first $1.15 \mu \mathrm{m}$ LPCVD polysilicon layer is deposited. The thickness of this layer determines the thickness of the flexible cross plate. On the polysilicon layer, a third $100 \mathrm{~nm}$ PECVD silicon oxide layer is deposited and patterned by BHF etching to define a layout of the cross-plate (b). A second $4.35 \mu \mathrm{m}$ LPCVD polysilicon layer is used to completely cover the patterned silicon oxide layer (c). Next, trenches are etched in the polysilicon using Deep Reactive Ion Etching (DRIE). The trenches are completely refilled with a low stress LPCVD silicon nitride layer. The silicon nitride layer is removed from the top surfaces by a maskless RIE (d). In a second DRIE step a motor layout is defined (e). The hidden silicon oxide layer serves as an etching mask allowing creation of the flexible plate and the stiff clamps in a single plasma etching step. Isolating bumps [7] are created on the backside of the clamps and the plate by a short isotropic etch of sacrificial silicon oxide followed by a deposition and directional removal of a low stress LPCVD silicon nitride layer. In the last fabrication step, the motor is released by removal of the sacrificial silicon oxide layer in $50 \% \mathrm{HF}$ (f).

\section{RESULTS}

A successfully fabricated 2-DOF shuffle motor, with all components, is shown in Figure 5. The whole motor, including four clamps and a cross plate fits in an area of 482 $\mu \mathrm{m} \times 482 \mu \mathrm{m}$. The motor is suspended with connection springs, which also provide driving voltage signal to the clamps and the plate. In this way, we avoid the use of underlying actuation electrodes allowing free motion of the motor over an unpatterned surface.

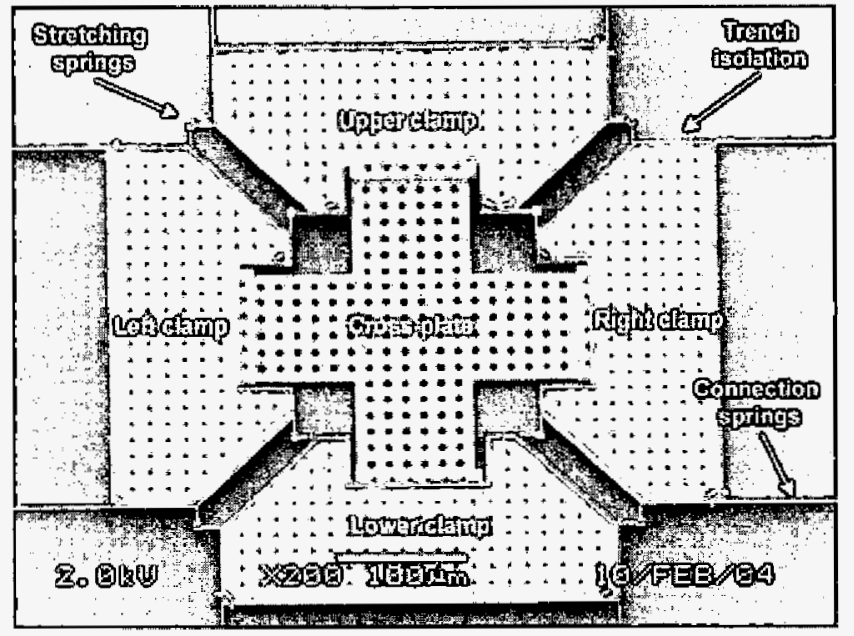

Figure 5: SEM micrograph of a fabricated 2DOF shuffle motor with all components.

Electrical isolation between the clamps and the plate was achieved by employing trenches refilled with a low-stress silicon nitride. The trench isolation, shown in Figure 6, allows independent biasing of each individual component. At the same time, a high stiffness and a large strength of the trench isolation assures mechanical integrity of the motor.

Small silicon nitride bumps, visible in Figure 6, are evenly divided over the backside surface of the clamps and the plate. During operation of the motor the isolating silicon nitride bumps only make contact with the silicon nitride coating on the wafer surface, reducing charge accumulation. The contact area is significantly reduced due to the rounded shape of the bumps. The reduction of the contact area prevents stiction of the motor during fabrication and operation. 


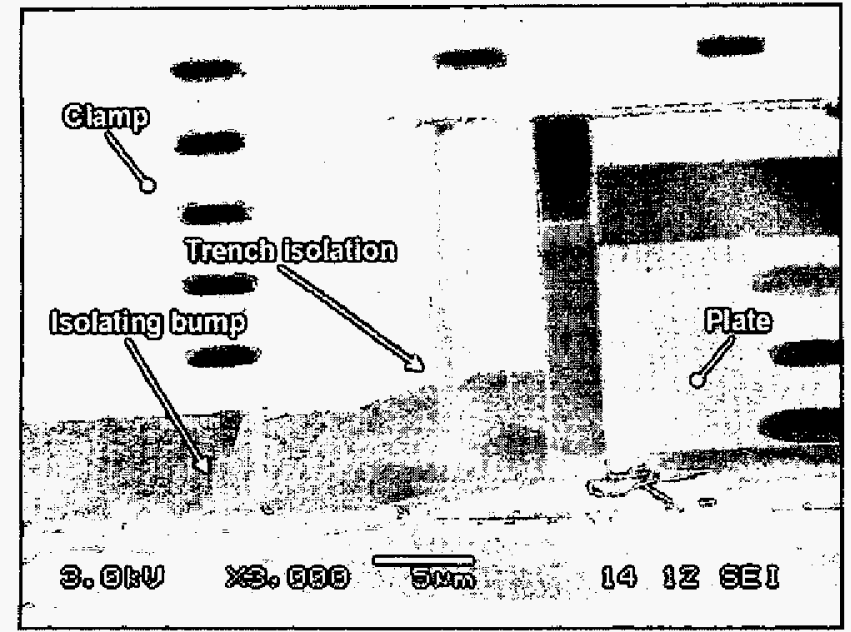

Figure 6: SEM micrograph showing a cross-section of a 2DOF shuffle motor before release step.

The fabricated motor is successfully operated and the inchworm motion is demonstrated in different stepping modes. An acquisition card and a high voltage amplifier with high slew rate are used for generation of the required voltage sequences. Actuation voltages up to $70 \mathrm{~V}$ are used in our experiments. Displacement of the motor is measured using an image processing technique. This technique, based on the Fourier transform, allows in-plane sub-pixel displacement measurements with a resolution of a few nanometers. The displacement range of the motor was 60 $\mu \mathrm{m}$ being only limited by the design layout. The maximum displacement was achieved using actuation voltages of $45 \mathrm{~V}$ on the plate and $36 \mathrm{~V}$ on the clamps. Using the springstiffness of the flexures a force of $0.64 \mathrm{mN}$ was calculated for the obtained displacement.

The velocity of the motor as a function of the cycling frequency, measured for two stepping modes, is given in Figure 7. The velocity measurement was performed on a motor operated with actuation voltage of $45 \mathrm{~V}$, both on the plate and the clamps. The measurement curves show a fairly linear increase of the velocity with increasing cycling frequency in the measured frequency region.

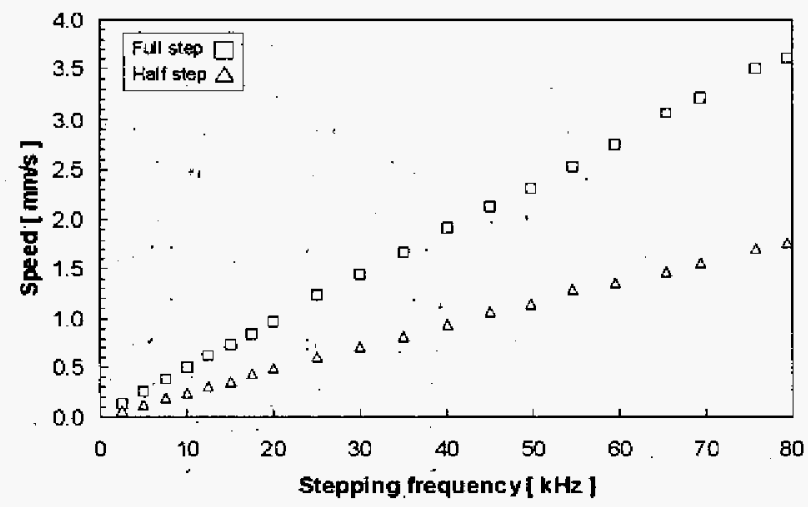

Figure 7: Measured speed versus stepping frequency for different stepping modes (clamp voltage $U_{c}=45 \mathrm{~V}$ and plate voltage $\left.U_{p}=45 \mathrm{~V}\right)$.
The slope of the curves is a measure for the average step size, which is $48 \mathrm{~nm}$ for the full step mode and $23.5 \mathrm{~nm}$ for the half step mode. The maximum cycling frequency of 80 $\mathrm{kHz}$ was only limited by the driving electronics.

The velocity of the 2-DOF shuffle motor depends not only on the cycling frequency and the stepping mode but also on the average step size. The step size of the motor is a function of the degree of plate deformation. A higher plate actuation voltage induces a larger plate deformation, resulting in a larger step size. Adjustable nanometerresolution steps ranging from $41 \mathrm{~nm}$ to $63 \mathrm{~nm}$, corresponding to plate voltages between $35 \mathrm{~V}$ and $70 \mathrm{~V}$, are measured.

\section{CONCLUSION}

The design, fabrication and characterization of an electrostatic linear inchworm micromotor with two translational DOF are presented. Large maneuverability, large force, high-resolution step, high speed and large displacement range are demonstrated.

\section{ACKWNOLEGMENTS}

The authors would like to thank M. de Boer, R. Sanders, T. Lammerink, N. Tas and $\mathrm{MESA}^{+}$clean room staff for their contribution to the fabrication and measurements. A part of the research, presented in this paper, is performed at Fujita Lab, University of Tokyo. All lab members are gratefully acknowledged. This work is a part of the research program on Micro Scanning Probe Array Memory ( $\mu$ SPAM) supported by the Dutch Technology Foundation (STW).

\section{REFERENCES}

[1] Bolks $M$ et al Micro Scanining Probe Array Memory (fSPAM) 2001 Proc. $2^{\text {nd }}$ PROGRESS workshop (Utrecht, The Netherlands)

[2] Vettiger $P$ et al 1999 Ultrahigh density, high-data-rate NEMS-based AFM data storage system Microelect. Eng. 46 11-17

[3] Carley L R et al 2000 Single-chip computers with microelectromechanical systems-based magnetic memory $J$. of Applied Physics 87 6680-5

[4] Tas N, Wissink J, Sander L, Lammerink T, Elwenspoek M 1998 Modeling, design and testing of the electrostatic shuffle motor Sensors and Actuators $A 70$ 171-8

[5] de Boer M P, Luck D L, Ashurst W R, Maboudian R, Corwin A D, Walraven J A, Redmond J M 2004 High performance surface-micromachined inchworm actuator $J$. Microelectormech. Syst. 13 63-74

[6] Sarajlic E, Berenschot E, Fujita H, Krijnen G, Elwenspoek M 2004 High performance bidirectional electrostatic inchworm motor fabricated by trench isolation technology submitted for Int. Conf. on Solid State Sensors and Actuators (Transducers 05) (Seoul, Korea)

[7] Sarajlic E, Berenschot E, Krijnen G, Elwenspoek M 2003 Versatile trench isolation technology for the fabrication of microactuators Microelect. Eng. 67-68 430-7 